\title{
On the environmental science and technology aesthetics and the innovation and development of modern science and technology
}

\author{
YUE Xiangyu ${ }^{1}$, YUE Youxi ${ }^{2}$ \\ ${ }^{1}$ School of Management and Engineering, Nanjing University; Nanjing Jiangsu; 210039; PR China \\ ${ }^{2}$ School of Chinese Literature and Journalism, Shandong University of Technology; Zibo Shandong; 255049; PR China
}

\section{Email address:}

yuexiangyu168@gmail.com(YUE Xiang-yu),youxiyue@aliyun.com(YUE You-xi)

\section{To cite this article:}

YUE Xiangyu, YUE Youxi. On the Environmental Science and Technology Aesthetics and the Innovation and Development of Modern Science and Technology. International Journal of Science, Technology and Society. Vol. 1, No. 2, 2013, pp. $42-47$.

doi: $10.11648 /$ j.ijsts.20130102.11

\begin{abstract}
The environmental science and technology aesthetics is a new interdisciplinary application aesthetic subject, which arises with the inspiration of contemporary ecological concepts, environmental ideas, and aesthetic thoughts. It is based on the theories of ecological ontology, ecological ethics and ecological value, and it is the perfect combination of science and technology and environmental aesthetics, and it is the science of the general laws in which science and technology are studied, innovated and developed in accordance with the principles of environmental aesthetics. It, from an aesthetic point of view, with the attitude, viewpoint and method of aesthetics, studies the environmental phenomena, environmental relationships and environmental laws between science and technology and nature, human and society. The environmental science and technology aesthetics is the concrete embodiment of the environmental ontology and aesthetics in the science and technology area. The environmental science and technology aesthetics has got rid of the Western traditional dualistic and opposing thinking mode, surpassed the modern tool reason and anthropocentrism, and promoted the environmental development of modern science and technology. It has great significance for us to maintain healthy, scientific and ecological development of modern science and technology, promote the construction of socialist environmental civilization, and build a beautiful China and a beautiful world. The environmental science and technology aesthetics is the concrete application of environmental aesthetics theory in science and technology area, while the innovation of environmental science and technology is the concrete embodiment of the environmental science and technology aesthetics in the modern science and technology innovation field. The environmental science and technology aesthetics is the important theoretical resource that guides the development of modern science and technology, and the only way to ensure the modern science and technology innovation develops healthily, scientifically and sustainably.
\end{abstract}

Keywords: Environmental Science and Technology Aesthetics, Science and Technology, Innovation, Development

\section{Introduction}

Since the 20th century, science and technology have developed and changed rapidly. The scientific and technological achievements in over ten decades of human beings have overtaken the combination of all the scientific and technological achievements in past centuries. The rapid development of science and technology has greatly changed the living circumstances of modern humans which to brought great benefits to our life and work. However, many scholars believe that it is like a double-edged sword. It brings convenience to mankind, but hurts humans in the mean time. For example, it caused serious human alienation and enormous damage to the natural ecological environment, so that it makes human spirit perplexed and confused in the state of alienation etc.[1](P1) Even some people think that the more advanced technology is, the greater alienation of human beings caused by it will be. Therefore, it thinks that the "embedded" systems, a representative of modern high-tech, will cause the most and largest alienation to human. However, from my opinion of view, what brings human the alienation is not the science and technology as a tool, but human themselves who use science and technology. More fundamentally speaking, it is the "culture" roots that mankind lives in. Therefore, to eliminate the so-called 
alienation brought by science and technology, first is to adjust the whole survival strategy of humanity, change human minds with a proactive cultural choice, thus completely change the current living condition of human. This choice of culture is ecological culture, the core of which is ecological aesthetics. Ecological Aesthetics is a panoramic cultural and scientific conception system covering all motion systems in the cosmos such as man and nature, man and society, man and man etc. Only by combining ecological aesthetics perfectly with scientific and technological innovation, taking the innovative way of "environmental science and technology aesthetics" can fundamentally eliminate the alienation which the science and technology have brought to mankind, revolutionize and improve the situation of human existence, as well as truly build a community of socialist ecological civilization, a beautiful China and even a beautiful world. Thus, environmental science and technology aesthetics has an important theoretical meaning and practical value to guide the innovation, development and improvement of modern science and technology.

\section{The Basic Connotation of Environmental Science and Technology Aesthetics}

What is "environmental science and technology aesthetics"? To understand the basic connotation of environmental science and technology aesthetics, we must first understand the "Ecological Aesthetics." Ecological Aesthetics is an interdisciplinary science based on the ecology and aesthetics. With the integral thinking of the ecosystem, ecology breaks the thinking tradition of subject-object dichotomy from the West, and views human race as a part of nature, thinking about in holistic nature, how humans develop in harmony with nature. Ecological Aesthetics is a new form of aesthetic theory which originates from the combination of ecology and aesthetics. It investigates aesthetic issues using ecological theory and method, important aspects of the ecology are absorbed into the aesthetic studies, and ecological way of thinking is applied to the field of aesthetics, thereby establishing the new form of aesthetic theory which adapts to social development. Its uniqueness lies in the organic integral way to look at aesthetic issues. Ecological beauty is the presentation of the aesthetic significance, it is realized by maintaining the ecological balance of the ecosystem of the entire world, including nature and human society, it suggests that man and nature have achieved a natural aesthetic state of coordinated development as well as coexistence and co-prosperity.

Environmental science and technology aesthetics mainly refers to the science arising from the combination of ecology, aesthetics and science and technology, it is an emerging interdisciplinary new aesthetic applications discipline which engenders with the inspiration of contemporary ecological concepts, environmental concepts and aesthetic concepts. Its theoretical foundation is based on Ecological Theory [2] (P52), Ecological Ethics [2] (P105) and Ecological Value [2] (P76). It is specific application of theoretical principles of ecological aesthetics in the areas of technological innovation, which means perfect combination of science and technology and environmental science and technology aesthetics, making the research, innovation and development of science and technology be in accordance with the principles of ecological aesthetics. It is the science which investigates the ecological phenomenon ecological relationships and ecological laws of science and technology as well as the nature, from the aspect of aesthetic with the aesthetic attitude, viewpoint and method. Environmental science and technology aesthetic is the concrete embodiment of ecological ontology and aesthetic in the technology sector. It has great innovation and pioneering. Environmental science and technology aesthetics not only focuses on qualitative (ie, the nature of existence) research of technology and nature, reconstruct the philosophical foundation during the inspection of inner life essence of science as well as technology and nature, and thus falls within the physical properties of category; but also comes with more aesthetic meaning, focusing on external form (namely, the existence of appearance) of science and technology and nature, and thus falls within the scope of phenomenology.

\section{Modern Technological Innovation and Environmental Science and Technology Aesthetics}

In face of the enormous destruction of human environment caused by the development of modern science and technology, technological innovation is turning to the exploration of mankind's future life, sustainable development and green peace instead of the simple pursuit of economic efficiency. From the 1992 Seville World Expo, the 1998 Lisbon World Expo to the 2000 Hanover World Expo, 2005 Aichi World Expo and 2010 Shanghai World Expo, they all identified the themes on global hot spots problems like the progress of human beings, science and technology and sustainable development etc. A number of eco-technologies responding to the theme of "science and technology" and "sustainable development" stood out and pointed out a new direction for the innovation and development of modern science and technology. Environmental science and technology follows the theoretical principles of environmental science and technology aesthetics, showing the respective attitude of human towards nature as well as placing the human "self-esteem" among the whole nature living harmoniously with it. This concept is undoubtedly affecting the technological aesthetics as well. It examines technology by putting it into a larger background making technological innovation and technological aesthetics ecological. Environmental science and technology aesthetics adheres to 
a kind of aesthetic concepts that completely different from which has supported technological development since the industrial civilization. Environmental science and technology is ecological not only in the internal structure and function, but also in the external image and human emotions in harmony with the surrounding environment. Environmental science and technology becomes a positive factor in maintaining the survival environment for human, rather than a destructive and negative factor. This is eco-technological beauty.

Environmental science and technology aesthetics provides theoretical support to modern eco-technology innovation to practice the sustainable development strategy, which makes technological innovation ecological and with full respect for nature, thus it can truly achieve the beauty of ecological science and technology. This is mainly manifested in the following aspects: ecological beauty of technological materials, ecological beauty in the progress of technological innovation, ecological beauty of technological images, ecological beauty of technology constructures, ecological beauty of scientific and technological functions, ecological beauty of technological products, ecological beauty of technology emotions and so on. The so-called ecological beauty of scientific and technological materials refers that the technological materials and ecological materials used in technological innovation are in the maximum use of natural materials, and green and environmental protective to the greatest extent, which try to make every kind of materials be green and be able to recycle and reuse, reducing the environmental load as much as possible. Ecological beauty in the progress of technological innovation refers that it should try to save energy, materials, manpower and material resources as many as possible in the process of technological innovation, and try not to produce harmful substances to the environment. Ecological beauty of technological images refers to the unity of consistency and harmony between the external morphology of technological innovational products and the surrounding environment. Ecological beauty of technology constructures indicates that the internal structure of scientific and technological achievements is as simple and applicable as possible, that every part is harmonious which achieve the optimal performance. Ecological beauty of scientific and technological functions means the optimized functionality of technological achievements, the least consumption, the maximum output, and the green output, which is environmentally benign and even beneficial to the environment. Ecological beauty of technological products points the largest ecologism to the production of scientific and technological achievements. Whether the features of the products or the exterior shape are harmonized with the surrounding natural environment. Ecological beauty of technology emotions refers to the healthy, active, and green scientific human emotions caused by the effect of technological achievements to people and the environment.

From the macro prospective of view of environmental science and technology aesthetics, modern technological innovation can make ecology achieve sustainable development and promote the harmonious unity between man and nature. Environmental science and technology aesthetics indicates that the coordination of ecological balance is of great essentiality. The harmonious coexistence of humans and nature through the ecologism of modern technology is a way to implement the eco-design and reflect the ecological beauty. There are several characteristics that make Environmental science and technology be different from other technologies: using the most advanced and greenest technologies and materials, expressing advanced green design concepts and considering successive use of scientific and technological achievements in the design period. The sustainable use of eco-technology is in unity of environmental science and technology aesthetics, which is the embodied practice of eco-technology aesthetics in the field of technological innovation. On one hand, ecological science and technology has aesthetic features as natural, simple and harmonious. The beauty of Environmental science and technology is a harmonious organic beauty of science and technology. It emphasizes the natural ecological beauty of science and technology and advocates simplicity and conciseness which brings people the enduring spirit of joy rather than the temporary visual shock. In terms of environmental science and technology aesthetics, ecological balance is a kind of harmony as well as a kind of order. It is through the natural and environmental protective materials as well as the simple technological structure and function which filled of wisdom though that reflects the natural and rustic characteristics of environmental science and technology aesthetics. On the other hand, eco-technology embodies the ecological sustainability. The eco-technological aesthetic standard develops from a single focus on the development of the beauty of scientific and technological forms to a new aesthetic standard that builds upon the ecological impacts, which is beyond the simple sensory appreciation, achieving harmony with nature in a spiritual dimension. The ultimate consideration is not only about the perfection of external aesthetic form in the current degree, but more about the computing and comparison between its ecological benefits and aesthetic cost. Environmental science and technology aesthetics can be said to be a functional aesthetics, a pragmatic aesthetics. It provides philosophical foundation for the ecologism of modern scientific and technological innovation and the practice of sustainable development. Environmental science and technology aesthetics can not only provide ideas and methods for eco-technological innovation, but also provide a reasonable scale for human to make use of nature, as well as guide people to correctly handle the relationship between the economic development and ecological protection, economic benefits and ecological benefits. As regard to ecological aesthetics of eco-technology, traditional scientific and technological design standards and aesthetic concepts tend to be broken. Scientists focus on technology's inner harmony of performance and organic synthetic expression of the technology and the structure. Areas of Concerning 
efficiency and technology in the range of terrestrial ecology and meeting the needs of environmental load reduction and sustainable development is the sole criterion to evaluate the beauty of modern ecological science and technology.

The core requirements of environmental science and technology aesthetics to ecological technological innovation are that first establish a reasonable civilized eco-relationship and structure of scientific and technological innovation, and second, solve the impacts of survival and life caused by technological factors from the perspective of ecological balance. The concept of "eco-technological design" and "green technological design" raised by environmental science and technology aesthetics lies its significance in providing new ideas to technological innovation, so that it enables people's minds back to nature and gains inspiration of technological innovation from nature, as well as reflects the damage to current ecological environment. To realize the significance of environmental science and technology aesthetics, we should link the natural environment to the modern science and technology which is developing rapidly now, creating a design that both fits the needs of people's material and spiritual life and possesses the natural shape of green technology and ecological harmony.

\section{Development and Prospects of Environmental Science and Technology}

Though nowadays humans' ecological consciousness is increasingly strong, modern technology has brought tremendous damage to the environment, which makes development of which becomes increasingly difficult. Some kinds of science and technology even went to the dead end. "Any circumstance hitting a limit will begin to change. Change will in turn lead to an unimpeded state, and then lead to continuity." Scientific and technological innovation will not have a healthy development until it is guided by ecological science aesthetics and follows the path of "eco-innovation". Technological innovation is turning to the exploration of mankind's future life, sustainable development and green peace from simply in the pursuit of economic benefits. Actually, to some extent, this is a "Return" of the spirit of traditional scientific and technological innovation.

In the age of traditional handicrafts design before the Industrial Revolution, labor productivity level was relatively low. The production was almost completely depend on manual operation. Nearly all materials used in production are natural materials, such as wood, stone, clay, leaves, weeds etc. In this way human activities made a comparatively small impact on the natural ecology. People not only were quite close to the nature but also rarely inflicted the destruction and pollution to it, which made human beings and the nature remain a relatively harmonious state. During this period, many technological innovations and features were derived from nature, such as in ancient
China, Sunju, recorded in "Kao Gong Ji ", fully embodies the harmony of technological innovation and eco-technology aesthetics. Sunju is the stent of ancient musical instruments like hanging bell, stones etc. The central crossbar is called Sun and two uprights are called Ju. It is both a practical device and a style of art. The shapes of Sunju are often closely related to vertebrates in nature. If a shape of grand sound animal is taken as a Zhongju with a bell with the same huge sound, the beauty of animal-shaped decoration and the beauty of sound practice will correspond to each other, casting beautiful reflections. In 1978, a zoomorphic Pan Ju, unearthed at the tomb of Zeng Houyi in Sui county, Hubei province, combined birds in the design of Ju's shape. More precisely, it was the kind of birds that had sharp mouths, open lips, small eyes, slender necks, short torsos and underdeveloped abdomens. They looked nimble with little strength which fitted for a lighter load force, together with the beautiful sound of the Pan, making a complement of visual and auditory appreciation and enhancing the appealing of whole work of art. The aesthetic meaning of eco-technology is accurately manifested in its appearance, internal structure and function. In addition, the productive significance of ecological science aesthetics can be proved on the product of some other ancient Chinese appliance. This could even more explain the ecological points and values of traditional Chinese culture. For instance, the shape and function of "Sharp-end Bottle", one of the typical devices in the culture of Yangshao, all show the concept of "If a bottle is empty, it will be lean. In the middle, it will be straight. If full, it will be overflowed." This suggests that people should properly handle the relationship between people and natural ecosystems and do not make it lose its balance. It is visible that the period of handicrafts is an important period of human civilization. Carrying the national culture, the various traditional products, created by artisans, indicate the idea that the Creator are trying to combine nature and products even in their consciousness, which keeps the harmony of human and nature.

In the West, past scientific and technological innovations also have ecological scientific and technological aesthetic thoughts. In the beginning of the Industrial Revolution, many famous designers saw the ugliness of industrial products, so they promoted the concept of antiindustrialization and proposed the concept of returning to nature. For example, Britain's John Ruskin advocates the idea of going back to nature and the naturalism of art designing. He emphasizes the curves and natural form, and is in the pursuit of the form of natural beauty as well as has an attempt to restore the old mode of production. Although this is contrary to the historical trend, it has played a positive and promoting role in the design and innovation of modern science and technology, which reflects people's longing to the combination of natural ecology and products as well as the important thought that human should be close to natural environment. Such idea is the basis for products having scientific and technological aesthetic significance.

In modern society, human's surrounding living 
environment suffers a huge damage, which affects the survival and development of mankind. Therefore people propose the concept of "ecology" which is gradually understood and accepted by us. When we saw the close correlation of human's destruction to the environment and the development of science and technology, we then produced a theoretical thinking of eco-technology aesthetics, and after that then provided the idea of creating the theoretical foundation of it. We emphasize that the concept of eco-technology aesthetics can be digested into modern scientific and technological innovations. During the cognition of the relationships between science and technology and the natural ecology, we realized that natural ecology must be an aesthetic value standard to the design and innovation of science and technology. Treating eco-technology as an aesthetic object and then producing different aesthetic ways is the significance of the beauty of it. These important significances are self-generating in Chinese ancient traditional appliances, which means that they are imprinted by the natural environmental science and technology on the date of production. With the development of the industrial age and the change of ecological concept, people have progressively had richer meanings to the technology's ecologicalization of products. Since we are keenly aware that the human is not a kind of independent creature but rather a section ring in the complex biological system, the relationships among humans and various ecological systems are inextricably linked. Thus, both in ancient, modern and future, humans must follow the laws of nature and the basic principles of eco-technology aesthetics in the process of technological innovation and other creatures, keeping harmonious co-exist of technology and nature, technology and society, technology and people, as well as man and nature.

In recent years, issues such as environmental protection, ecological balance as well as psychological ecological requirements have aroused people's attention. As a result, eco-technological innovation and aesthetic issues are also increasingly paid much attention. Many designers indicates that technological innovation should not only meet the needs of people's material and spiritual life, but also pursue some design ideas with more positive meaning. Like bionic design which regards the products as a carrier making human and nature be more close to each other. When we see a eco-technological product, through its form and function, we can realize the idea of eco-technological and aesthetic design and creation in it, which makes technological products and environmental science and technology aesthetics complement each other. Such eco-technological beauty is not a general sense of natural beauty. Natural beauty is the aesthetic value of nature itself, while eco-technological beauty is the natural ecological value of science and technology highlighted in the eco-technological innovation. To achieve the transformation of technological products from scientific beauty to eco-technological beauty, people as technology innovators who is an indispensable factor. Ecol-technological beauty emphasizes inherent natural character of products through the their appearances. This kind of character becomes the "junk" for human emotions to be relied on. It is loaded with people 's emotions. This is the communication between human and natural ecology rather than which between man and technological products. Behind the nature of eco-technological products implies the ecological concepts abiding together by people and nature, reflecting the close relationship between man and nature to share a harmonious common prosperity.

Future technological innovation and development will inevitably follow the eco-technology aesthetics which is also the only way. The innovational results of ecological science and technology will reflect the aesthetic character of natural ecology, no matter from the choice of materials, the external form of technological products, or its internal function and structure. Even in the beginning of product design, it is necessary to take into account the issue of recycling products. Either recycle them, or digest then in nature non-pollutionally. Ecological and technological innovation should not only consider the economic benefits of the products, but consider the social and ecological benefits, paying attention to their natural attributes and ecological aesthetic properties. The eco-technological product consider its natural attributes and ecological aesthetic characteristics on the appearance, so that people can feel and enjoy the natural beauty as well as arise the desire and conservation to natural ecology when they are using the product. This kind of innovation and development of eco-technological aesthetic products follow a path of green technology which tallies with human's environmental science and technology aesthetics. Only then will it not give greater pressure to the ecological environment. Humans can then share a harmonious common prosperity with nature and live a low-carbon life in this high speed society, truly benefiting our children and grandchildren.

\section{Concluding Remarks}

In summary, ecological science aesthetics is a new interdisciplinary aesthetic application discipline arising under an inspiration of contemporary ecological concepts, environmental concepts and aesthetic concepts. The theoretical foundations of which are ecological ontology, ecological ethics and ecological value. It is a science of general laws which perfectly combines scientific technology and ecological aesthetics, enabling scientific technology to research, innovate and develop with the principles of ecological aesthetics. It is a science studying ecological phenomena, ecological relationships and ecological laws between science and technology and the nature from the aesthetic point of view with the aesthetic attitudes, viewpoints and methods. Ecological science aesthetics is the embodiment of ecological ontology and aesthetics in the technology sector. It gets rid of Western traditions of dualistic thinking, which goes beyond the modern instrumental rationality and anthropocentrism, and also promotes ecological development of modern 
technology. It has important significance for keeping the health of modern science and technology and the sound development of science, promoting the construction of socialist ecological civilization as well as building a beautiful China and a beautiful world. Ecological science aesthetics is the concrete application of ecological aesthetic theory in the field of scientific and technological innovation, while eco-technological innovation is the embodiment of ecological science aesthetics in the field of modern scientific and technological innovation. Ecological science aesthetics is an important theoretical resource to guide the development of modern science and technology. It is also the only way to ensure the health of modern scientific and technological innovation, science and sustainable development.

\section{Introduction to the Author}

Yue Xiangyu (1992- ), who is a male, Han nationality, was born in Weifang, Shandong, with BA, Nanjing University, mainly engaged in electrical information and automation, aesthetics and other aspects of learning and research, specializing in modern industrial embedded control systems, networked control systems, intelligent control. He presided over one national college student science and technology innovation project, taking part in the research on one "Eleventh Five-Year Plan" education and science key project of the Education Ministry, and one soft science research project of Shandong Province. He has 12 science and technology papers published in Chinese and oversea academic journals. He has won the 1st scholarship of China, the 1st scholarship of people, the 1st and the 2nd prizes of China Education Robotics Competition, the 1st prize of China Mathematical Modeling Contest, the top award of scientific research achievement of Nanjing University, two China science and technology patents and the honorary title, "three-good-student" of Jiangsu Province. In addition, as a representative of Nanjing University, he went to the National University of Singapore to participate in an academic exchange program. Address: School of Management and Engineering, Gulou Campus of Nanjing University, 22, Hankou Avenue, Gulou District, Nanjing, Jiangsu Province, PR China; Zip: 210039.

Yue Youxi, male, Han nationality, post-doctor, visiting scholar of Harvard University, was born in Weifang Shandong province in June, 1967, who is a professor, graduate student tutor of the School of Chinese Literature and Journalism, Shandong University of Technology \&. Shandong Research Base for the Soft Sciences of Eco-culture and Recycling Economy, and principally good at teaching and doing research on the literature theories, aesthetics, environmental culture, and so on.

\section{References}

[1] Yue Youxi. Pursuing Poetic Inhabitation [M]. Beijing: People's Publishing House 2009.

[2] Yue You-xi. The Environmental Aesthetics [M]. Beijing: People's Publishing House, 2007.

[3] Hu Jintao, the Chinese Communist Party eighteen report [A], Xinhua November 8, 2012. 